\title{
Conhecimento e socialização organizacional: processo sociocultural para a inovação na indústria de eletro-eletrônicos de Garça/SP
}

\author{
Conocimiento y socialización organizational: proceso sociocultural para la innovación \\ en la industria electro-electrónica de Garça, São Paulo \\ Knowledge and organizational socialization: sociocultural process for innovation \\ in the electronics industry of Garça, São Paulo
}

\section{Cássia Regina Bassan de Moraes, Luana Maia WoidA, Andréia de Abreu, Deise Deolindo SiLva}

Faculdade de Tecnologia de Garça, Av. Presidente Vargas, 2331, CEP: 17.400-000, Garça, São Paulo, Brasil, crbassan@gmail.com, luanamwoida@yahoo.com.br, andreiabreu11@gmail.com, deisedeolindo@hotmail.com

\begin{abstract}
Resumen
Se discute el papel de la socialización organizacional en los procesos de creación innovativa de conocimiento en las industrias del sector electro electrónico de la ciudad de Garça, São Paulo. Se utilizó una metodología cualititiva, basada en la aplicación in situ de cuestionarios con escalas de Likert a los gerentes, y la utilización de instrumentos estadísticos para identificar correlaciones entre las variables. Los resultados confirman la contribución de los procesos de socialización a la construcción de conocimiento para la innovación en las industrias de electro electrónicas.

Palabras clave: Socialización organizacional. Gestión del conocimiento. Innovación. Industrias eléctricas y electrónicas. Brasil. Garça (São Paulo).
\end{abstract}

\section{Conhecimento e Organizações}

A participação dos indivíduos na produção de conhecimento direcionado para os objetivos organizacionais depende dos processos socioculturais que determinam as maneiras pelas quais o conhecimento é produzido, compartilhado e usado. Isso porque, os processos socioculturais são responsáveis por internalizar no indivíduo condições, modelos e expectativas de um grupo quanto ao comportamento e a forma correta de perceber e resolver problemas.

Os indivíduos presentes nos níveis gerenciais, entendidos como os cargos responsáveis por planejar, organizar, dirigir e controlar as tarefas e desempenho dos subordinados, geralmente são responsáveis por desenvolver conhecimentos para intermediação ou aplicação nos níveis estratégico, tático e operacional. Cabe a esse nível efetivar a inovação na organização. Ou seja, são responsáveis por fornecer informações importantes sobre a execução das tarefas aos

\begin{abstract}
The role of organizational socialization processes of innovative knowledge creation in the electroelectronic industrial sector of Garça city in São Paulo is discussed. A qualititive methodology based on the on-site application of a Likert-scale questionnaire to the managers and the utilization of statistical tools to identify correlations among variables was used. The results confirm the contribution of the socialization process to knowledge construction for supporting innovation in the electro-electronic industries sector.
\end{abstract}

Keywords: Orgazational socialization. Knowledge management. Innovation. Electric and electronic industries. Brasil. Garça (São Paulo).

novatos (Miller y Jablin, 1991; Morrison, 1993 apud Bauer y Green, 1998).

A socialização organizacional é um processo necessário em toda a extensão da estruturura organizacional, norteando o comportamento das pessoas, uma vez que se trata da inserção que visa a instrução e a internalização de dada realidade.

Posto isso, a socialização organizacional é um processo sociocultural fundamental para a atuação dos níveis gerenciais, pois auxilia inserindo não apenas os gerentes no contexto de produção de conhecimento, como também ajuda o nível gerencial nesse processo de inserção dos subordinados.

O conhecimento produzido, compartilhado e usado na organização para a inovação depende de como os comportamentos são estimulados e internalizados. Nem sempre as determinações e normas são cumpridas, bem como a realidade posta é considerada correta pelo indivíduo, sendo necessário por meio de técnicas de sociali- 
zação, a devida incorporação pelas pessoas de parâmetros adequados em seus comportamentos. A socialização contribui diretamente para isso, porque conduz o sujeito na adoção dos parâmetros considerados corretos na organização.

Assim, o conhecimento deve se desenvolver na presença de processos socioculturais capazes de estimular maior participação e adesão dos indivíduos na realidade da organização. Todavia, deve-se assinalar que nem todos os presentes na estrutura organizacional participam igualmente da inovação, alguns atuam em seu planejamento, enquanto outros colocam-na em prática. Dessa forma, o tipo de socialização organizacional usado como subsídio da inovação depende da posição hierárquica ocupada, conforme assinala a literatura (Woida, Moraes y Abreu, 2013), pois o tipo de conhecimento acessado, produzido e usado é diferente.

Nesse caso, corrobora-se com a definição de Valentim (2008, p. 19) sobre conhecimento, entendendo-o como o produto de um sujeito cognitivo, que internaliza informações para produzir conhecimento individual, que por sua vez, influi sobre o conhecimento coletivo, do qual partiu para a construção individual. Inclui-se nessa perspectiva, o que assinala Davenport e Prusak (1998, p. 6) pois consideram o conhecimento uma mistura de experiências, de valores, de informação contextualizada e da própria experiência, podendo ser encontrado na forma de documentos, de repositórios, rotinas e práticas, processos e normas da organização.

Por sua vez, a inovação é considerada como algo novo na percepção dos indivíduos. Como prediz a OCDE (2004), a inovação se divide em duas categorias: a tecnológica de produto e a de processo. A primeira se divide ainda em novos produtos (apresentando características e usos diferentes dos existentes) e produtos melhorados (o produto existe, porém apresenta atualização ou evidente aperfeiçoamente em relação ao original). No segundo, a inovação tecnológica de processo enfatiza a adoção de métodos novos ou melhorados.

Assim, a inovação implica na participação direta de indivíduos comprometidos e preparados para construírem e aplicarem ideias novas aos produtos e processos das organizações, tratandose de item indispensável para a atuação das indústrias de eletro-eletrônicos da cidade de Garça-SP, por ser um setor altamente tecnológico, cujo fulcro de competitividade está na inovação.

O objetivo da pesquisa exposta neste trabalho é diagnosticar os fatores envolvidos na socialização organizacional que contribuem para os processos de criação de conhecimento para a inovação nas indústrias do ramo eletroeletrônico da cidade de Garça-SP.

Neste trabalho, a abordagem qualitativa se sobressai, com aplicação de instrumentos estatísticos visando identificar correlações entre as variáveis, especialmente entre a prática de comportamentos informacionais resultados da socialização vinculados a presença da inovação em indústrias do setor de eletro-eletrônicos. Definiram-se como sujeitos de pesquisa os gerentes que atuam nessas indústrias. Os questionários foram estruturados no formato da escala de Likert, uma vez que além das correlações, foi relevante identificar a tendência positiva ou negativa nas respostas dos sujeitos. Foram entrevistados 53 sujeitos para a pesquisa, sendo 20 na empresa PPA - Portas e Portões Automáticos Ltda; 17 na empresa RCG Tecnologia Eletromecânica Ltda; 16 na empresa SPC - Eletromatic Controle e Proteção Ltda.

\section{Socialização organizacional}

Um dos questionamentos condutores desse trabalho foi: como conseguir inovação por meio das pessoas? Contudo, outros questionamentos foram emergindo, tais como: quem são os indivíduos que participam da inovação e como contribuem? Assim, tornou-se evidente a necessidade de compreender quais são os aspectos envolvidos na socialização e sua relação com a construção do conhecimento e a inovação. Entretanto, supõe-se que os fluxos de informação importantes para a idealização da inovação, bem como de sua implementação se originam dos níveis superiores da estrutura.

Dessa forma, os aspectos relevantes para o estudo se concentraram sobre identificar as técnicas de socialização e sua relação com a construção de conhecimento para a inovação.

As técnicas de socialização são importantes para instituir e propagar uma realidade considerada correta na organização. Compreende-se a socialização a partir das duas abordagens principais: processo de imposição e processo de incorporação e adequação. Nesse caso, tanto a realidade pode ser previamente determinada (Berger y Luckmann, 2004), como adaptada para os interesses do indivíduo novato (recémchegado) que internaliza ou modifica o conhecimento adquirido conforme os próprios interesses e julgamento (Borges y Albuquerque, 2007). Isso significa que a realidade posta pela organização é tanto condutora do comportamento como também uma construção social da realidade. 
A socialização organizacional tem como marco de seus primórdios, a Década de 1960 , sendo seus principais idealizadores teóricos Van Maanen e Schein (1979) e Jones (1986) apud Borges e Albuquerque (2007). No início o interesse desses estudos era compreender a formação e influência sobre os comportamentos, facilitando a aprendizagem e a internalização de conhecimentos sobre determinada organização.

Compreende-se a socialização como um momento de adequação ou transição, no qual assimilam-se conhecimentos sobre o grupo de tarefas e sobre o ambiente social de trabalho, incluindo os comportamentos dos membros já socializados anteriormente (Van Maanen y Schein, 1979, apud Bauer y Green, 1998). Porém, de acordo com Berger e Luckman (2004), os indivíduos possuem pouca autonomia para mudança ou adequação sobre o conhecimento ou realidade institucionalizada.

Além disso, a socialização é o processo pelo qual ocorre a incorporação da doutrina e a formação de indivíduos entrantes na organização (Schein, 1968). Nesse caso, a socialização também pode ser considerada treinamento.

A literatura registra precedentes sobre a relação da socialização com a informação. No início, os estudos visavam associar a socialização à busca de informação. Uma das abordagens era sobre a informação como redutora de incerteza (Berlyne, 1960; Lanzetta, 1971, apud Morrinson, 1993; Berger, 1979) e a segunda, inseria a informação como indispensável para executar as tarefas na organização (Graen, Orris y Jonhson, 1973; Jablin, 1984 apud Morrinson, 1993; Dirsmith y Covaleski, 1985). Dessa forma, o tipo de informação determina o tipo de socialização pelo qual o indivíduo deve ser submetido.

Considerando esse aspecto, é pressípuo que informações necessárias para a inovação se vinculem a gerência e a uma técnica específica ou grupo de técnicas de socialização. Nesse caso, além da necessária presença de compartilhamento e de comportamentos de busca, destacam-se tipos de socialização organizacional definidos pelo tipo de informação requerida para o trabalho e o desempenho dos indivíduos: informação técnica, necessária para executar as tarefas; informação de referencia, sobre a demanda dos papéis e das expectativas tanto sobre a execução como sobre o status do papel (Miller y Jablin, 1991, apud Morrison, 1993); informação normativa, atitudes e comportamentos esperados e aprovados (Louis, 1990, apud Morrison, 1993; Comer, 1991); informação de feedback, que revela o desempenho do indivíduo, e a forma de avaliação e percepção de terceiros; e informação relacional, concentra-se apenas sobre as percepções e avaliação por outras pessoas do novato (Ashford, 1986, apud Morrison, 1993).

As táticas de socialização organizacional sugeridas por Van Maanen (1996, p. 47-60) são: formais, informais, individuais, coletivas, sequenciais, não-sequenciais, fixas, variáveis, por concurso, competição, em série, isolada, despojamento e investidura.

\section{Resultados e discussão}

A análise realizada sobre os dados foi obtida com base no Teste Qui-quadrado, buscando verificar a independência. Destacam-se neste artigo três correlações entre as questões do instrumento de pesquisa. Na primeira, a socialização (treinamento) oferecido aos funcionarios para obter melhor relacionamento com os pares de trabalho vincula-se ao treinamento para obter o compartilhamento do conhecimento. Essa dependência foi comprovada pelo Teste Quiquadrado ao nível de significância de $5 \%$. Nesse caso a literatura indica que a socialização possui funções específicas na organização, seja para inserir, seja para instruir sobre o que realmente é considerado importante (Schein, 1968). Além disso, essa correlação indica que o processo de socialização usado avança sobre questões vinculadas à obtenção de parâmetros sobre o relacionamento na organização, uma vez que o resultado dos relacionamentos deve ir além de cumprir as tarefas específicas de cada cargo. Muitas organizações atuais buscam na construção de conhecimento, uma maneira de galgar vantagens competitivas, assumindo que as contribuições individuais são fundamentais para isso e são obtidas em processos de compartilhamento. Por sua vez, estes advém de relacionamentos construídos e estimulados pela organização.

Uma segunda comparação mostrou que o treinamento oferecido a todos os funcionários promove a busca e a construção de conhecimento, sinalizando os efeitos da socialização organizacional. O nível de significancia para essa correlação também foi de $5 \%$, comprovando a dependência. Como elementos importantes, destacam-se que o processo de socialização utiliza uma tática coletiva (Van Maanen, 1996) bem como se concentra em estimular que as informações técnicas se sobressaiam, pois a partir delas o conhecimento organizacional específico para o negócio é aperfeiçoado. Apesar da tática de socialização ser coletiva, o resultado é a construção de conhecimento individual, além de mostrar que as organizações pesquisadas con- 
duzem a socialização de forma a incorporar o maior número de pessoas em seus objetivos.

Ao nível de significância de $5 \%$ verificou-se dependência entre o treinamento oferecido aos funcionários para promover a busca e construção de conhecimento e o treinamento que estimula a melhoria do trabalho individual. Nesse ponto, ressalta-se fundamental para a inovação que os indivíduos executem o trabalho sempre visando melhorias. A literatura sugere que as táticas individualizadas estimulam mais a criatividade, conduzindo à inovação (Jones, 1986 apud Borges y Albuquerque, 2007). Entretanto, as melhorias são conseguidas em processos de adaptação às necessidades e formas de pensar individuais, sendo posteriormente repassadas ao coletivo.

Por fim, a socialização organizacional praticada nas indústrias pesquisadas auxilia nos contextos coletivos e individuais, indicando que ora visa apenas instruir sobre tarefas específicas, ora visa transmitir conhecimentos sobre a organização e sobre as formas pelas quais as pessoas devem colaborar para buscar, construir e compartilhar o conhecimento. A socialização é um processo de inserção praticado pelas indústrias, de formas diferentes em cada nível da estrutura organizacional, cujo intuito é ajudar os indivíduos a incorporarem a nova realidade, conforme assinalaram os gerentes que responderam ao instrumento de pesquisa. Assim, os fatores da socialização que contribuem para a inovação porque estimulam as pessoas a criarem e a compartilharem conhecimento são tanto as táticas individuais como coletivas, cujas fontes de informação usadas são internas e externas à organização.

\section{Notas}

(1) Pesquisa financiada pelo Conselho Nacional de Desenvolvimento Científico e Tecnológico (CNPq), Chamada $\mathrm{MCTI} / \mathrm{CNPq} / \mathrm{MEC} / \mathrm{CAPES} \mathrm{N}{ }^{\circ}$ 18/2012.

\section{Referencias}

Abreu, Andréia de; Moraes, Cássia Regina Bassan de, \& Woida, Luana Maia (2013). O processo de socialização organizacional: a inserção de usuários no contexto de acesso à informação para a competitividade. // CETAC. MEDIA. (Org.). Globalização, ciência, informação: atas VI Encontro Ibérico EDICIC 2013. Porto: Faculdade de Letras da Universidade do Porto - CETAC.MEDIA, 2013. 1394-1411.

Bauer, Talya N.; Green, Stephen G. (1998). Testing the combined effects of newcomer information seeking and manager behavior on socialization. // Journal of Applied Psychology. 83:1, 1998. 72-83.
Berger, C. R. (1979). Beyond initial understanding: Uncertainty, understanding, and the development os interpersonal relationships. // H. Giles, R. N. Clair. Language and social pychology. Oxford: Basil Blackwell, 1979. 112-134.

Berger, P. L., Luckmann, T. (2004). A construção social da realidade: tratado de sociologia do conhecimento. $24 \mathrm{ed}$. Petrópolis: Vozes, 2004.

Berlyne, D. E. (1960). Conflict, arousal and curiosity. New York: MacGraw-Hill, 1960.

Borges, L. O., Albuquerque, F. J. B. (2004). Socialização organizacional. // Zanelli, J. C., Borges-Andrade, J. E., Bastos, A. V. B. (Org.). Psicologia, organizações e trabalho no Brasil. Porto Alegre: Artmed, 2004. 332-356.

Comer, D. R. (1991). Organizational newcomers' acquisition of information from peers. // Management Communication Quarterly. 5 (1991) 64-89.

Davenport, T. H.; Prusak, L. (1998). Conhecimento empresarial: como as organizações gerenciam seu capital intelectual. Rio de Janeiro: Campus, 1998.

Dirsmith, M. W., Covaleski, M. A. (1985). Informal communications, nonformal communications, and mentoring in public accounting firms. // Accounting, Organizations, and Society. 10 (1985) 149-169.

Graen, G., Orris, J. B., Johnson, T. W. (1973). Role assimilation processes in a complex organization. // Journal of Vocation Behavior. 3 (1973) 395-420.

Jablin, F. M. (1984). Assimilating new members into organizations. // Bostrom, R. Communication yerbook.. Beverly Hills: Sage. 8 (1984) 594-626.

Jones, G.R. (1986). Socialization tactics, self efficacy, and newcomers' adjustments to organizations. // Academy of Management Journal. 29 (1986) 262-279.

Louis, M. R. (1990). Acculturation in the workplace: newcomers as lay ethnographers. // Schneider, B. Organizational climates and cultures. San Francisco: Jossey-Bass, 1990. 85-127.

Miller, V. D., \& Jablin, F. M. (1991). Information seeking during organizational entry: influences, tactics, and a model of the process. // Academy of Management Review. 16 (1991) 92-120.

Morrison, Elizabeth Wolfe (1993). Newcomer information seeking: exploring types, modes, sources, and outcomes. // Academy of Management Journal, 36:3 (1993) 557-589.

OCDE-Manual de Oslo (2004). Proposta de diretrizes para coleta e interpretação de dados sobre inovação tecnológica. Finep - tradução português, 2004.

Schein, E.H. (1968). Organizational socialization and the profession of management. Industrial Management Review. 9 (1968) 1-16.

Valentim, M. L. P. (2008) Informação e conhecimento em organizações complexas. // Valentim, M. L. P. (Org.). Gestão da informação e do conhecimento no âmbito da ciência da informação. São Paulo: Polis/Cultura Acadêmica, 2008. 11-26.

Van Maanen, J. (1996). Processando as pessoas - estratégias de socialização organizacional. // Fleury, M. T. L.; Fischer, R. M. Cultura e poder nas organizações. São Paulo: Atlas, 1996. 45-62.

Van Maanen, J., Schein, Edgar H. (1979). Toward a theory of organizational socialization. // Staw, B. M. Research in organizational behavior. Greenwich: CT JAI Press. 1 (1979) 209-264. 


\section{Anexo: entrevista aplicada nas empresas}

1. Recebe orientações sobre as tarefas que executará na empresa. 2. Recebe orientações sobre quem serão os colegas de trabalho no mesmo departamento.

3. Recebe orientações sobre quem serão os colegas de trabalho em departamentos ou setores que deverá manter contato.

4. Recebe orientações sobre a história da empresa.

5. Recebe orientações sobre as pretensões de expansão e crescimento da empresa.

6. As informações e conhecimentos são adquiridos somente pela experiência.

7. O treinamento é feito em etapas e quando o grupo de tarefas é complexo.

8. Realiza-se treinamento para conseguir a aceitação de tecnologias de informação

9. Realiza-se treinamento para conseguir o uso de tecnologias de informação

10. Os funcionários recebem treinamento quando uma nova tecnologia associada ao trabalho que executa é adquirida.

11. Os funcionários recebem incentivo para buscar e efetivar treinamentos e formação em ambientes como escolas e faculdades.

12. Todos os funcionários recebem treinamento na empresa.

13. Todos os funcionários do departamento que trabalho recebem treinamento.

14. Os funcionários recebem treinamento individual.

15. Os funcionários recebem treinamento coletivo.

16. Os funcionários recebem treinamento para conseguirem novos cargos na empresa.

17. O treinamento é oferecido continuamente na empresa.

18. Os funcionários recebem treinamento para adquirir competências profissionais.

19. Os funcionários recebem treinamento para se relacionarem melhor com os colegas de trabalho.

20. Os funcionários recebem treinamento para conseguirem compartilhar conhecimento com os demais colegas.

21. Os funcionários recebem informações para conhecerem a política da empresa

22. Os funcionários recebem informações para compreenderem como e o que é comunicado dentro da empresa.

23. Os funcionários recebem informações para conhecerem os objetivos e valores da empresa.

24. Os funcionários recebem informações para conhecerem a história da empresa.

25. O treinamento oferecido aos funcionários facilita a apropriação dos valores defendidos pela empresa.

26. O treinamento oferecido aos funcionários promove comprometimento com as tarefas e objetivos da empresa.

27. O treinamento oferecido aos funcionários promove a busca e construção de conhecimento.

28. O treinamento oferecido facilita a identificação dos funcionários que são fontes de informação importantes.

29. O treinamento oferecido estimula a melhoria do trabalho executado individualmente.

30. O treinamento oferecido estimula a melhoria do trabalho executado coletivamente

31. Os funcionários recebem informações dos superiores (chefes, gerentes, supervisores, líderes)

32. Os funcionários adquirem informações em manuais disponibilizados pela empresa.

33. Os funcionários recebem treinamento para buscar e acessar a informação na empresa.

34. Os funcionários usam a informação adquirida em treinamento para a tomada de decisão.

35. Os funcionários usam a informação adquirida em treinamento para criar conhecimento.

36. Os funcionários compartilham a informação com os colegas.

37. Os funcionários compartilham a informação com o superior (chefe, gerente, supervisor, líder).

38. Existe estímulo para buscar informações para conhecer o próprio trabalho.

39. Existe estímulo para buscar informações para melhorar a interação social com os colegas de trabalho.

40. Existe estímulo para buscar informações no ambiente externo da empresa.

41. Existe estímulo para buscar feedback junto ao superior (chefe, gerente, supervisor,

líder) sobre o próprio desempenho.

\begin{tabular}{|c|c|c|c|c|}
\hline 1 & 2 & 3 & 4 & 5 \\
\hline $\begin{array}{l}\text { Nenhuma } \\
\text { vez }\end{array}$ & $\begin{array}{c}\text { Pouquíssimas } \\
\text { vezes }\end{array}$ & $\begin{array}{l}\text { Poucas } \\
\text { vezes }\end{array}$ & $\begin{array}{l}\text { Muitas } \\
\text { vezes }\end{array}$ & $\begin{array}{l}\text { Muitíssimas } \\
\text { vezes }\end{array}$ \\
\hline & & & & \\
\hline & & & & \\
\hline & & & & \\
\hline & & & & \\
\hline & & & & \\
\hline & & & & \\
\hline & & & & \\
\hline & & & & \\
\hline & & & & \\
\hline & & & & \\
\hline & & & & \\
\hline & & & & \\
\hline & & & & \\
\hline & & & & \\
\hline & & & & \\
\hline & & & & \\
\hline & & & & \\
\hline & & & & \\
\hline & & & & \\
\hline & & & & \\
\hline & & & & \\
\hline & & & & \\
\hline & & & & \\
\hline & & & & \\
\hline & & & & \\
\hline & & & & \\
\hline & & & & \\
\hline & & & & \\
\hline & & & & \\
\hline & & & & \\
\hline & & & & \\
\hline & & & & \\
\hline & & & & \\
\hline & & & & \\
\hline & & & & \\
\hline & & & & \\
\hline & & & & \\
\hline & & & & \\
\hline & & & & \\
\hline & & & & \\
\hline & & & & \\
\hline
\end{tabular}

Enviado: 2014-04-01. Segunda versión: 2014-06-11.

Aceptado: 2014-06-23.

Moraes, Cássia Regina Bassan; Woida, Luana Maia; Abreu, Andréia; Silva, Deise D. Conhecimento e socialização organizacional: processo sociocultural para a inovação na indústria de eletro-eletrônicos de Garça/SP. // Ibersid. 8 (2014) 91-95. ISSN 1888-0967. 
